\title{
FLUTUAÇÃO POPULACIONAL DE ESPÉCIES DE CIGARRINHAS TRANSMISSORAS DA CLOROSE VARIEGADA DOS CITROS (CVC) EM VIÇOSA - MG ${ }^{1}$
}

\author{
DIERLEI DOS SANTOS 2 , DALMO LOPES DE SIQUEIRA³, MARCELO COUTINHO PICANÇO ${ }^{4}$
}

\begin{abstract}
RESUMO - Os objetivos deste trabalho foram avaliar a presença e a dinâmica populacional, ao longo do ano, de cigarrinhas transmissoras da CVC, no município de Viçosa - MG. As coletas foram realizadas no período de agosto de 2003 a julho de 2004, com auxílio de armadilhas adesivas amarelas e com rede entomológica (puçá). Das onze espécies comprovadamente capazes de transmitir a CVC, foram encontradas nove. O número total de cigarrinhas capturadas foi de 2.966, sendo 2.805 nas armadilhas e 161 no puçá. As três espécies da família Cicadellidae mais expressivas em número de insetos foram Bucephalogonia xanthophis (36,6\% capturados na rede entomológica e 34,7\% na armadilha), Dilobopterus costalimai (9,9\% capturados na rede entomológica e 11,3\% na armadilha) e Acrogonia citrina (6,8\% capturados na rede entomológica e 7,4\% na armadilha). No mês de novembro, foram coletadas 378 cigarrinhas, correspondendo ao mês de maior incidência, sendo que 292 dessas foram coletadas na primeira quinzena, concorrendo para um pico populacional. Não houve diferença significativa entre o número médio de cigarrinhas coletadas no interior e na periferia da área.
\end{abstract}

Termos para indexação: CVC, Xylella e cigarrinhas.

\section{POPULATION DYNAMICS OF SHARPSHOOTERS SPECIES TRANSMITTER OF CITRUS VARIEGATED CHLOROSIS (CVC) IN VIÇOSA - MG}

\begin{abstract}
The objectives of this work were to evaluate the presence and the population dynamics of Citrus CVC transmitter sharpshooters along the year in the municipality of Viçosa- MG. The sharpshooter populations were monitored in the period of August 2003 to July 2004 using yellow adhesive traps and entomologic nets. During this period 2966 sharpshooters were captured and identified. Among eleven species known as CVC transmitters, nine were found in the region. The three most expressive species in number found were Bucephalogonia xanthophis (36,6\% captured in entomological net and 34,7\% in the trap), Dilobopterus costalimai (9,9\% captured in entomological net and $11,3 \%$ in the trap) and Acrogonia citrina (6,8\% captured in entomological net and 7,4\% in the trap). The largest incidence of sharpshooters occurred in November ( 378 insects), with the population reaching the pick in the first fifteen days of the month (292 insects). There was not significant difference among the medium number of sharpshooters collected in the interior and in the periphery of the area.
\end{abstract}

Index terms: CVC, Xylella and sharpshooters.

\section{INTRODUÇÃo}

A clorose variegada dos citros (CVC), causada pela bactéria Xylella fastidiosa (Wells et al., 1987) e transmitida por cigarrinhas é uma doença que foi identificada pela primeira vez em 1987, em municípios do noroeste paulista (Rossetti, 1990; Rossetti \& De Negri, 1990). Posteriormente, foi constatada em vários estados do Brasil, inclusive em algumas regiões de Minas Gerais; entretanto, não foi encontrado na literatura nenhum trabalho relatando a ocorrência de cigarrinhas transmissoras da CVC em Minas Gerais e a sua dinâmica populacional ao longo do ano. Essas informações são importantes, pois auxiliam na avaliação do risco de disseminação da doença em regiões onde ainda não foi constatada, principalmente na Zona da Mata Mineira, onde existe um pólo de produção de mudas de citros a céu aberto.

Onze espécies de cigarrinhas da família Cicadellidae são comprovadamente capazes de transmitir a CVC ao se alimentarem de plantas doentes. São elas: Dilobopterus costalimai, Acrogonia citrina, Oncometopia facialis, Bucephalogonia xanthophis, Plesiommata corniculata, Macugonalia leucomelas, Homalodisca ignorata, Parathona gratiosa, Ferrariana trivittata, Acrogonia virescens e Sonesimia grossa (Paiva et al., 1996; Yamamoto, 1996; Yamamoto \& Gravena, 2000; Yamamoto et al., 2002, Fundecitrus, 2003).

A característica marcante utilizada por taxonomistas para diferenciar insetos da família Cicadellidae das demais famílias da ordem Hemiptera é a presença de uma ou mais fileiras de espinhos nas tíbias posteriores (Barror, 1992).

Existem vários métodos de amostragem da população de cigarrinhas, sendo que os mais práticos e baratos são as armadilhas adesivas amarelas e a rede entomológica (puçá), porém o primeiro método vem apresentando melhores resultados (Fundecitrus, 2003).
A avaliação de cigarrinhas transmissoras da $\mathrm{CVC}$ em regiões produtoras de mudas de citros, principalmente em céu aberto, é importante, pois caso seja constatada sua presença, há necessidade de verificar se elas são portadoras da bactéria Xylella fastidiosa ou se as plantas presentes no local de avaliação estão contaminadas. Caso positivo, os sistemas de produção de borbulhas e mudas terão de ser modificados para o sistema de Produção de Mudas Certificadas de Citros (IMA, 2000), que prevê a produção de borbulhas e de mudas em telados à prova de insetos, como já é obrigatoriamente usado em São Paulo (Revista do Fundecitrus, 2002).

Este trabalho teve como objetivo avaliar se as espécies transmissoras da Clorose Variegada dos Citros (CVC) estão presentes na Zona da Mata Mineira e acompanhar a flutuação populacional ao longo do ano.

\section{MATERIALEMÉTODOS}

O experimento foi realizado em viveiro de produção de mudas de citros, localizado no Pomar do Fundão, pertencente à Universidade Federal de Viçosa, Departamento de Fitotecnia (Setor de Fruticultura).

A área é de aproximadamente 1,5 ha, na qual estão plantadas treze cultivares de citros: laranjas 'Pêra-Rio', 'Valência', 'Natal', 'Bahia', 'Baianinha', 'Serra D’água', 'Lima', 'Campista'[Citrus sinensis (L.) Osbeck]; Tangor 'Murcote' (híbrido), tangerinas 'Poncã' (Citrus reticulata Blanco) e 'Rio'(Citrus deliciosa Ten.); limas ácidas 'Galego' [Citrus aurantifolia (Christm.) Swing.] e 'Tahiti' (Citrus latifolia T.), enxertadas sobre o limoeiro 'Cravo' (Citrus limonia .Osb.).

O trabalho iniciou-se em agosto de 2003 e terminou em julho de 2004, com um total de 24 avaliações. A coleta, contagem e identificação das cigarrinhas foram realizadas quinzenalmente, e a troca de armadilha

\footnotetext{
(Trabalho 015/2005). Recebido: 13/01/2005. Aceito para publicação: 01/08/2005.

2 Aluno de graduação do curso de Agronomia da Universidade Federal de Viçosa, Departamento de Fitotecnia, Viçosa- MG, 36570-000, Bolsista do CNPq, E-mail: dierlei@pop.com.br.

${ }_{3}^{3}$ Eng. Agr., DS, Professor Adjunto da Universidade Federal de Viçosa , Departamento de Fitotecnia, Viçosa- MG, 36570-000, E-mail: siqueira@ufv.br.

${ }^{4}$ Eng. Agr., DS, Professor Adjunto da Universidade Federal de Viçosa, Departamento de Biologia Animal, Viçosa- MG, 36570-000, E-mail: picanco@ufv.br.
} 
foi feita mensalmente.

A presença e a flutuação populacional das cigarrinhas foram avaliadas por amostragens quinzenais, utilizando os dois métodos (armadilhas adesivas amarelas e rede entomológica).

Foram utilizadas 40 armadilhas com dimensões de 10 x $11 \mathrm{~cm}$, sendo que vinte e três foram distribuídas em toda a periferia, visando a detectar a possível movimentação de cigarrinhas das áreas adjacentes ao viveiro, e dezessete foram distribuídas em pontos eqüidistantes no interior da área.

Cada armadilha permaneceu no campo por um período de 30 dias. No final da primeira quinzena, todos os insetos foram retirados das armadilhas, contados e identificados. Após a remoção dos insetos com pinça, as armadilhas continuaram no campo para a captura da segunda quinzena, na qual foi adotado o mesmo procedimento de coleta da primeira, seguido da substituição das armadilhas

As armadilhas foram fixadas em estacas de bambu, a uma altura de 1,70 $\mathrm{m}$, para que ficassem acima da altura média das plantas, permitindo fácil acesso às cigarrinhas. Para cada coleta, foi contado o número de cigarrinhas aderidas à superfície (frente e verso) de cada armadilha. Para a coleta com a rede entomológica (puçá), foram amostrados 20 pontos no interior da área, distribuídos uniformemente, sem considerar variações nas cultivares. Em cada ponto, foram amostradas 05 plantas, totalizando 100 plantas por amostragem.

Os procedimentos de identificação e cálculos foram os mesmos tanto para método de coleta das armadilhas adesivas amarelas quanto para o método da rede entomológica.

Com o objetivo de avaliar, estatisticamente, se houve migração de cigarrinhas da periferia para o interior da área no período estudado, foi utilizado o teste de $\mathrm{t}$, a $5 \%$ de probabilidade, entre as médias das cigarrinhas das armadilhas localizadas na periferia e no interior.

Calcularam-se a freqüência e a constância das espécies da família Cicadellidae coletadas nas armadilhas adesivas amarelas e na rede entomológica. Freqüência corresponde a porcentagem de indivíduo de cada espécie em relação ao total de indivíduos coletados. Para o cálculo da constância, empregou-se a fórmula $\mathrm{C}(\%)=\mathrm{P} / \mathrm{N}$. 100, onde $\mathrm{C}=$ constância das espécies, expressa em porcentagem; $\mathrm{P}=$ número de coletas contendo a espécie; $\mathrm{N}=$ número de coletas realizadas

As espécies foram classificadas como constantes quando estavam presentes em mais de $50 \%$ das coletas; acessórias quando presentes entre 25 e $50 \%$ das coletas; acidentais quando presentes em menos de 25\% das coletas (Silveira Neto et al., 1976).

As cigarrinhas da família Cicadellidae foram identificadas até a categoria de espécie, e as de outras famílias foram denominadas "outras cigarrinhas".

O trabalho de identificação foi realizado com auxílio de microscópio estereoscópio dotado de equipamento fotográfico digital

A identificação de insetos de algumas espécies foi realizada com o auxílio do Fundecitrus (Fundo de Defesa da Citricultura) e a UFPR (Universidade Federal do Paraná).

\section{RESULTADOS E DISCUSSÃO}

Das onze espécies de cigarrinhas com potencial de transmissão da bactéria Xylella fastidiosa, nove foram verificadas, sendo elas: $B$. xanthophis; D. costalimai; A citrina; O. facialis; M. leucomelas; $H$. ignorata; P. corniculata; P. gratiosa; F. trivittata (Tabela 2).

Na rede entomológica, apenas a espécie $B$. xanthophis foi constante, as espécies $D$. costalimai, A. citrina, $O$. facialis foram acessórias e $M$. leucomelas, $P$. gratiosa e $H$. ignorata foram de ocorrência acidental (Tabela 1). As espécies B. xanthophis, D. costalimai, $A$. citrina, $O$. facialis e $H$. ignorata foram constantes nas armadilhas adesivas amarelas, enquanto $P$. corniculata, $F$. trivittata e $M$. leucomelas foram acessórias e $P$. gratiosa acidental (Tabela 2).

Houve predominância da espécie B. xanthophis (Figura 2). Segundo Lopes (1999), essa espécie está presente na maioria dos pomares de citros em formação, sendo a mais eficiente transmissora de
TABELA 1 - Número, freqüência e constância das cigarrinhas coletadas na rede entomológica - Agosto de 2003 a julho de 2004.

\begin{tabular}{lccc}
\hline \multirow{2}{*}{ Espécies identificadas } & \multicolumn{3}{c}{ cigarrinhas } \\
\cline { 2 - 4 } & $\begin{array}{c}\text { Total } \\
\left(\mathbf{n}^{\mathbf{0}} \mathbf{)}\right.\end{array}$ & $\begin{array}{c}\text { Frequência } \\
\mathbf{( \% )}\end{array}$ & $\begin{array}{c}\text { Constância } \\
\text { das espécies }\end{array}$ \\
\hline B. xanthophis & 59 & 36,6 & constantes \\
D.costalimai & 16 & 9,9 & acessórias \\
A. citrina & 11 & 6,8 & acessórias \\
O. facialis & 8 & 5,0 & acessórias \\
H. ignorata & 1 & 0,6 & acidentais \\
M. leucomelas & 1 & 0,6 & acidentais \\
P. gratiosa & 1 & 0,6 & acidentais \\
Outros cicadellideos & 19 & 11,8 & acidentais \\
Outras cigarrinhas & 45 & 28,1 & constantes \\
TOTAL & 161 & 100,0 & - \\
\hline
\end{tabular}

TABELA 2 - Número, freqüência e constância das cigarrinhas coletadas nas armadilhas amarelas - Agosto de 2003 a julho de 2004.

\begin{tabular}{lccc}
\hline \multirow{2}{*}{ Espécies identificadas } & \multicolumn{3}{c}{ cigarrinhas } \\
\cline { 2 - 4 } & $\begin{array}{c}\text { Total } \\
\left(\mathbf{n}^{\mathbf{0}} \mathbf{.}\right)\end{array}$ & $\begin{array}{c}\text { Frequência } \\
\mathbf{( \% )}\end{array}$ & $\begin{array}{c}\text { Constância } \\
\text { das espécies }\end{array}$ \\
\hline B. xanthophis & 973 & 34,7 & constantes \\
D. costalimai & 318 & 11,3 & constantes \\
A. citrina & 207 & 7,4 & consantes \\
O. facialis & 101 & 3,6 & constantes \\
H. ignorata & 49 & 1,7 & constantes \\
M. leucomelas & 24 & 0,9 & acessórias \\
P. gratiosa & 5 & 0,2 & acessórias \\
P. corniculata & 8 & 0,3 & acidentais \\
F. trivittata & 8 & 0,3 & acidentais \\
Outros cicadellideos & 208 & 7,4 & constantes \\
Outras cigarrinhas & 904 & 32,2 & constantes \\
TOTAL & 2805 & 100,0 & - \\
\hline
\end{tabular}

$X$. fastidiosa. A probabilidade de transmissão da bactéria por um único indivíduo da espécie $B$. xanthophis é de apenas $11,7 \%$.

Esses dados indicam a importância do controle das cigarrinhas em viveiros de produção de mudas a céu aberto, borbulheiras e pomares em formação, para minimizar o risco de aquisição da bactéria pelas cigarrinhas e infecção das plantas.

Foram contados 973 exemplares na armadilha adesiva amarela de B. xanthophis (Tabela 2), número que supera em $34 \%$ a soma dos insetos das demais espécies. Na rede entomológica, foram capturados 59 exemplares (Tabela 1), representando 36,6\% do total. Essas informações mostram a clara predominância de B. xanthophis, pois as demais espécies transmissoras representaram apenas $25,7 \%$ nas armadilhas e 23,5\% na rede entomológica (Tabelas 1 e 2).

A armadilha amarela é mais eficiente do que a rede entomológica para a captura de cigarrinhas. Nas armadilhas, foi capturado maior número de exemplares de cada espécie e algumas espécies que não foram capturadas com a rede entomológica, como $P$. corniculata e $F$. trivittata (Tabelas 1 e 2).

Nota-se que as espécies mais capturadas (B. xanthophis, $D$. costalimai e $A$. citrina) representaram, respectivamente, $36,6 \%, 9,9 \% \mathrm{e}$ $6,8 \%$ do total de insetos capturados durante o experimento, na rede entomológica. Nas armadilhas amarelas, as porcentagens foram de $34,7 \%$, $11,3 \%$ e $7,4 \%$. Observa-se que, nos dois métodos de captura, a ordem de freqüência foi a mesma para as três espécies predominantes.

Apesar de os dois métodos de coleta apresentarem freqüências semelhantes, o mesmo não aconteceu com a constância. Enquanto as três espécies, $B$. xanthophis, $D$. costalimai e $A$. citrina, se apresentaram constantes na armadilha amarela, apenas $B$. xanthophis foi constante na rede entomológica. Esse fato ocorreu devido à baixa eficiência de coleta pela rede entomológica, mencionada anteriormente, visto que as cigarrinhas estavam presentes na época de coleta, pois foram capturadas pelas armadilhas amarelas.

Observou-se que, quando o número de cigarrinhas coletadas 
foi baixo, ocorreu maior variação nos valores da freqüência e constância das espécies de cigarrinhas entre os dois métodos (Tabelas 1 e 2).

No mês de novembro, foram coletadas 378 cigarrinhas, correspondendo ao mês de maior incidência, sendo que 292 dessas foram coletadas na primeira quinzena, concorrendo para um pico populacional (Figuras 1 e 3). Esses resultados, provavelmente, foram devidos às condições climáticas favoráveis ao inseto nesse período.

Para 11 dos 12 meses de coletas, o número de cigarrinhas da segunda quinzena de cada mês foi inferior ao da primeira quinzena (Figura 1). A justificativa para esse fato, provavelmente, está na perda de atratividade das armadilhas, uma vez que ficaram expostas ao sol, sendo observada redução gradativa na intensidade da coloração amarela. Esse fato indica que as substituições das armadilhas devem ocorrer a intervalos menores. Apenas em junho, a coleta da segunda quinzena foi superior à da primeira (Figura 1).

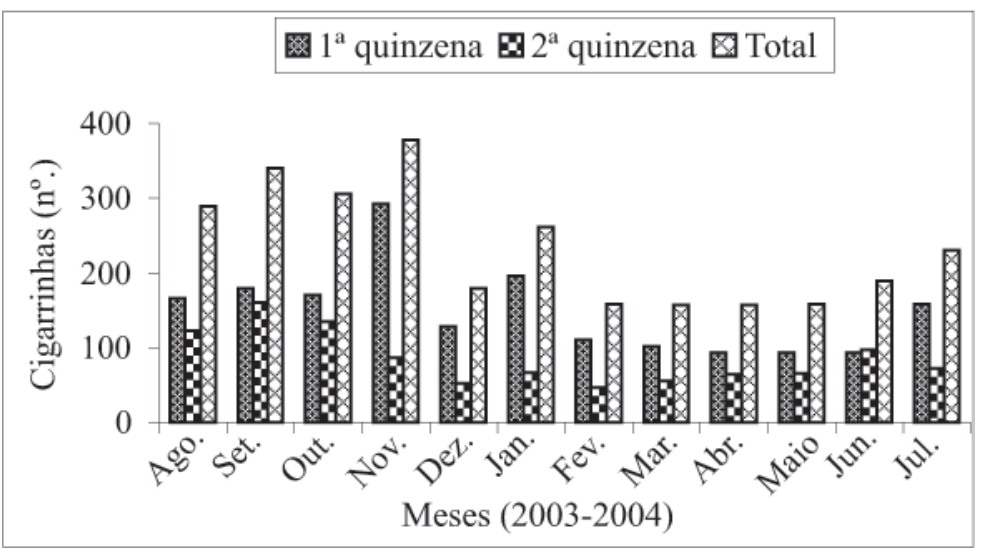

FIGURA 1 - Número de cigarrinhas, coletadas pelo método da armadilha adesiva amarela, na primeira quinzena, segunda quinzena e total do mês.

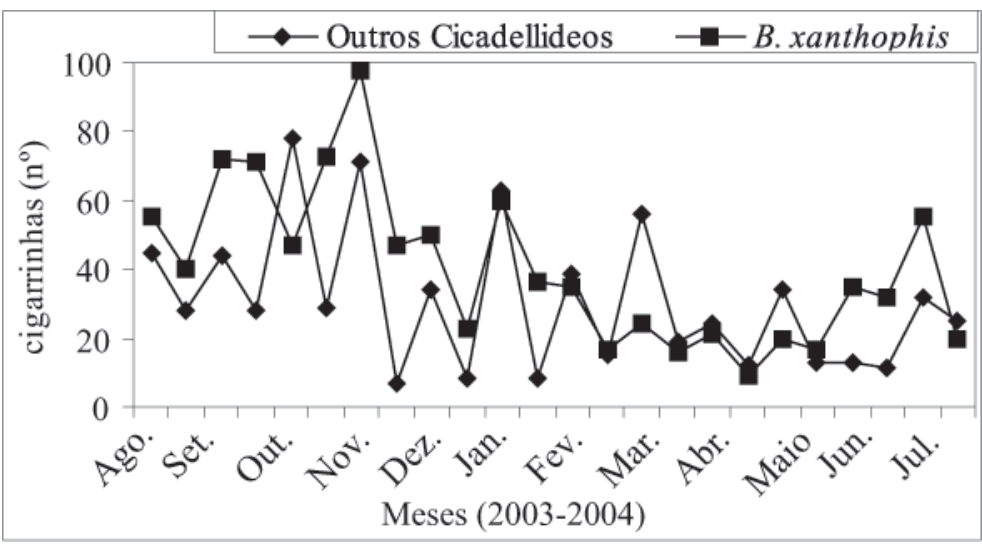

FIGURA 2 - Flutuação populacional de B. xanthophis comparando com a soma de outros Cicadellidios transmissores da CVC, capturados em armadilha adesiva amarela, no período de agosto de 2003 a julho de 2004.

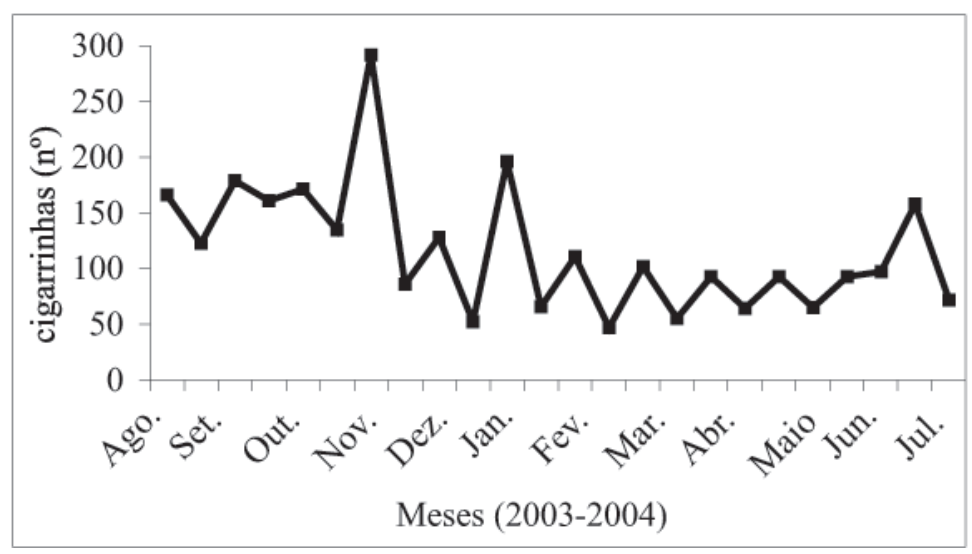

FIGURA 3 - Flutuação populacional de todas as cigarrinhas coletados com armadilha adesiva amarela no viveiro de citros da UFV, no período de agosto de 2003 a julho de 2004.

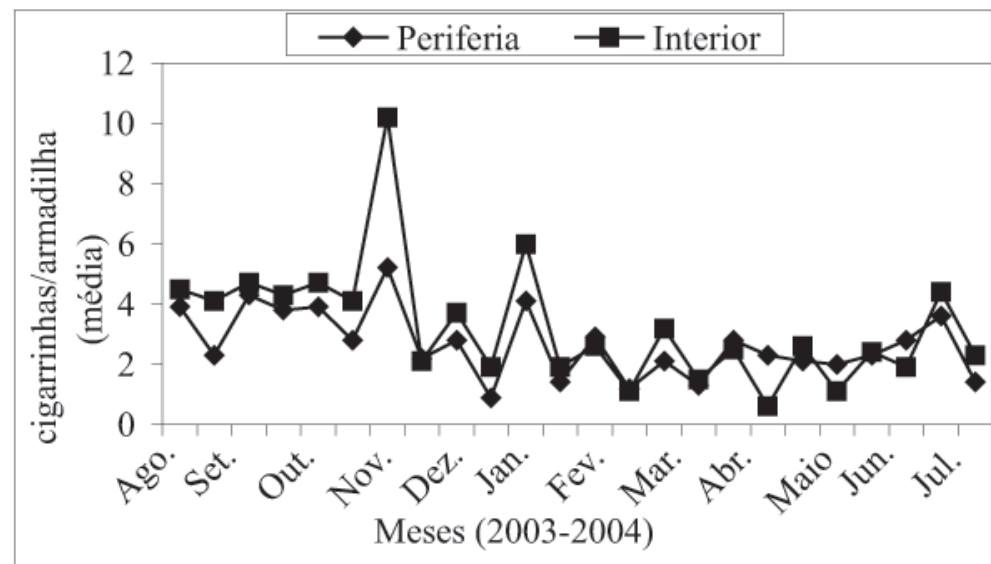

FIGURA 4 - Flutuação populacional de todas as espécies de cigarrinhas (média) coletadas com armadilha adesiva amarela no interior do viveiro e na periferia, no período de agosto de 2003 a julho de 2004

O teste de $\mathrm{t}$, a $5 \%$ de probabilidade, demonstrou que não foi significativa a migração de cigarrinhas da periferia para o interior da área ao longo do período estudado, embora nos primeiros meses de coletas as médias no interior tenham sido superiores (Figura 4). Possivelmente, as cigarrinhas entraram diretamente no viveiro, sem passar pelas armadilhas periféricas, visto que o viveiro se encontra numa área isolada por mata nativa. Ou mesmo por estarem se reproduzindo livremente no interior da área devido à não-realização de controle químico desse inseto, ressaltando também a presença de ervas daninhas nas entrelinhas. Segundo Hopkins (1995), as cigarrinhas vetores da X. fastidiosa possuem uma ampla gama de plantas hospedeiras.

Comparando esses resultados com os obtidos por Yamamoto (1998) em São Paulo, foram observadas diferenças na flutuação populacional e espécies predominantes. Atribui-se essa diferenciação a fatores como fenologia do hospedeiro e diferenças climáticas ao longo do ano. Entretanto, de modo geral, o pico populacional ocorreu na primavera/verão em todos os experimentos.

\section{CONCLUSÕES}

1. Foi constatada a presença, em Viçosa - MG, de nove das onze espécies de cigarrinhas da família Cicadellidae consideradas transmissoras da CVC, sendo que as espécies capturadas com maior número de representantes foram B. xanthophis, D. costalimai e $A$. citrina, tanto na armadilha adesiva amarela como na rede entomológica.

2. A armadilha adesiva amarela é mais eficiente do que a rede entomológica na captura de cigarrinhas, mas pode perder sua atratividade com o tempo de exposição.

\section{REFERÊNCIAS}

BARROR, D. J.; FREPLEHORN, C.A.; JOHNSON, N. F. An introduction to the study of insects. $6^{\text {th }}$ ed. Orlando, FL.: Saunders College Publishing, 1992. 875p.

FUNDECITRUS.. Departamento Científico: apresenta informações sobre cigarrinhas. Disponível em $<$ http://fundecitrus.com.br/ cigar.html $>$. Acesso em: 22 abr. 2003.

HOPKINS, D. L. Xylella fastidiosa. In: SINGH, U.S.; SINGH, R. P.; KOHMOTO, K. (Ed.). Pathogenesis end host specificity in plant diseases. Tarrytown: Elsevier Science, 1995. v.1, cap. 7, p. 185-197.

IMA- Instituto Mineiro de Agropecuária. Produção de mudas certificadas de citros. Portaria n.363/2000 de 11 de janeiro de 2000. Belo Horizonte, 11 jan. 2000.

LOPES, J.R.S. Estudos com vetores de Xylella fastidiosa e implicações no manejo da clorose variegada dos citros. Laranja, Cordeirópolis, v. 20, n. 2, p. 329-344, 1999.

PAIVA, P.E.B.; SILVA, J.L.; GRAVENA, S.; YAMAMOTO, P.T. 
Cigarrinhas de xilema em pomares de laranja do Estado de São Paulo. Laranja. Cordeirópolis: v. 17, n. 1, p. 41-54, 1996.

REVISTADO FUNDECITRUS. Viveiros: produção a céu aberto em MG preocupa técnicos. Araraquara: Fundecitrus, v. 14, n. 109, 2002. $15 \mathrm{p}$.

ROSSETTI, V.; DE NEGRI, J. Clorose variegada dos citros: Revisão. Laranja. Cordeirópolis, v. 11, n. 1, p. 1-14, 1990.

ROSSETTI, V. Resultados preliminares de estudos sobre uma nova anormalidade dos citros observada nos Estados de São Paulo e Minas Gerais. Summa Phitopathologica, Botucatu, v.16, p. 1-13, 1990.

SILVEIRA NETO, S.; NAKANO, O.; BARBIN, D.;VILA NOVA, N.A. Manual de ecologia dos insetos. São Paulo: Editora Agronômica Ceres, 419p. 1976.

YAMAMOTO, P. T. Cigarrinhas em citros no Estado de São Paulo. Laranja. Cordeirópolis: v. 17, n.1, p. 237-239, 1996.
YAMAMOTO, P. T. Espécies e flutuação populacional de cigarrinhas e psilídios (Hemiptera) em pomares cítricos. 1998. 112f. Tese (Doutorado em Agronomia)-Faculdade de Ciências Agrárias e Veterinárias, Universidade Estadual Paulista, Jaboticabal, 1998.

YAMAMOTO, P. T.; GRAVENA, S. Espécies e abundância de cigarrinhas e psilídios (Homoptera) em pomares cítricos. Anais da Sociedade Entomológica do Brasil, Londrina, v.29, n. 1, p.155-162, 2000.

YAMAMOTO, P. T.; ROBERTO, S.R.; PRIAJÚNIOR, W.D.; FELIPPE, M.R.; FREITAS, E.P. Espécies e flutuação populacional de cigarrinhas em viveiro de citros, no município de Mogi Guaçu-SP. Revista Brasileira de Fruticultura, Jaboticabal: v. 24, n.1, p. 389394, 2002.

WELLS, J.M.; RAJU, B.C.; HUNG, H.Y.; WEINSBERG, W.G.; MANDELCO-PAUL, L.; BRENNER, D.J. Xylella fastidiosa gen. nov. sp. nov.: Gram-negative, xylem-limited fastidious plant bacteria related to Xanthomonas spp. International Journal of Systematic Bacteriology, Washington, v.37, p.136-143, 1987. 